\section{Lipids mediate podocyte damage}

In diabetic kidney disease (DKD), the link between diabetes and damage to podocytes is unclear. Mitochondrial dysfunction caused by lipid accumulation in podocytes might increase their susceptibility to injury, as reported by Alessia Fornoni, Sandra Merscher, Flavia Fontanesi and colleagues.

"We had previously shown that glomerular expression of phospholipid-transporting ATPase ABCA1 was lower in patients with DKD than in healthy controls, and that reducing Abca1 expression in mouse podocytes caused cholesterol accumulation," explains Merscher. To assess the potential contribution of $A B C A 1$ to kidney disease in the context of diabetes, the researchers induced a podocyte-specific deletion of Abca1 in diabetic ob/ob mice. The loss of Abca1 was accompanied by an increase in albuminuria and mesangial expansion, a reduction in the number of podocytes and podocyte foot processes, and increased cholesterol ester in the kidney.

The researchers also noted mitochondrial swelling in podocytes from $\mathrm{Abca1}^{-/-} \mathrm{ob} / \mathrm{ob}$ mice. Targeting Abca1 with small interfering RNA in podocytes in vitro not only reduced oxidative phosphorylation and $\mathrm{O}_{2}$ consumption but also significantly increased cardiolipin content. Cardiolipin is a mitochondrial phospholipid that accumulates in cells from patients with loss-of-function $A B C A 1$ mutations. Treating $d b / d b$ diabetic mice with $A 30$, a drug that increases ABCA1 expression, specifically reduced docosahexaenoic acid-rich cardiolipin species and significantly improved the kidney phenotype. Similarly, elamipretide, a drug that inhibits cardiolipin peroxidation, prevented DKD progression in ob/ob mice. "ABCA1 deficiency leads to cardiolipin accumulation and mitochondrial dysfunction," adds Fontanesi. "These results are the first to link podocyte lipid metabolism to mitochondrial dysfunction in DKD."

"Our next step is to identify and validate drugs that prevent cardiolipin peroxidation and/or esterified cholesterol accumulation," remarks Fornoni. "We are designing a phase II clinical trial to test a small molecule inducer of ABCA1 in patients."

Monica Wang

ORIGINAL ARTICLE Ducasa, G. M. et al. ATP-binding cassette A1 deficiency causes cardiolipin-driven mitochondrial dysfunction in podocytes. J. Clin. Invest. https://doi.org/10.1172/ JCl125316 (2019)

\title{
CD9 boosts PEC glomerular invasion
}

Crescentic glomerulonephritis (CGN) and focal segmental glomerusclerosis (FSGS) are characterized by glomerular injury caused by invading parietal epithelial cells (PECs). Now, Carole Henique, Pierre-Louis Tharaux and colleagues report that CD9 contributes to this pathogenic process.

"Having observed that heparin-binding epidermal growth factor-like growth factor (HB-EGF) was critical to mediate severe CGN, we decided to study molecular partners of HB-EGF, such as CD9," explains Tharaux. "In this new study, when we measured RNA and protein expression it became clear that de novo expression of CD9 occurs primarily and robustly in PECs in various unrelated extracapillary lesions in mice and humans." Staining of patient samples showed that CD9 colocalized with integrin $\beta 1$ (ITGB1) and CD44 in extracapillary GN but not in in non-proliferative GN or in healthy kidneys.

Global $C d 9^{-/-}$mice were protected from CGN, but not mice in which $C d 9$ was only deleted in platelets, which express high levels of CD9. Irradiated wild-type mice reconstituted with $C d 9^{-/-}$bone marrow were also susceptible to CGN. "However, selective
CD9 deficiency in PECs prevented a large part of glomerular failures in FSGS and crescentic GN models, showing that PECs are not just bystanders but are truly pathogenic," notes Henique.

PECs in which $C d 9$ was knocked down proliferated less in response to HB-EGF or platelet-derived growth factor (PDGF) than scramble controls. Using a microfluidic system, the researchers showed that $C d 9$ knockdown also prevented migration towards a PDGF gradient - loss of Cd9 led to impaired phosphorylation of PDGFR $\beta$, EGFR and focal adhesion kinase 1 (FAK). "CD9 might lower the response threshold of PECs to factors released by the diseased capillary tuft, driving coordinated directional migration, adhesion and sclerosis," remarks Tharaux.

"Our work indicates that the abnormal behaviour of PECs is a clear therapeutic target for FSGS and CGN," adds Henique.

Monica Wang

ORIGINAL ARTICLE Lazareth. $\mathrm{H}$. et al. The tetraspanin CD9 controls migration and proliferation of parietal epithelial cells and glomerular disease progression. Nat. Commun. 10, 3303 (2019)

\section{URAEMIC TOXINS}

\section{Remote sensing and signalling in the kidney controls microbial metabolite levels}

Several gut microbiota-derived metabolites accumulate in the blood of patients with chronic kidney disease (CKD). A recent study by Rosalinde Masereeuw and colleagues reports that kidney proximal tubule cells sense elevated levels of these metabolites and activate a signalling pathway that enhances their secretion.

"Understanding the process of renal secretion of endogenous waste products might lead to the identification of novel leads for the treatment of kidney disease," explains Masereeuw. "We focused on organic anions, with indoxyl sulfate - a microbial metabolite of dietary tryptophan - as a marker uraemic toxin." Indoxyl sulfate is very toxic at high concentrations and is associated with comorbidities and clinical outcomes in patients with CKD.

The researchers previously showed that a high-protein diet increased the renal excretion of indoxyl sulfate in healthy humans and rodents. Now, they demonstrate that indoxyl sulfate induces the expression and function of organic anion transporter 1 (OAT1) in renal proximal tubule cells. "We found that binding of indoxyl sulfate to the EGF receptor leads to downstream activation of the AhR signalling pathway and induction of OAT1, which facilitates removal of the metabolite," says Masereeuw. "This pathway explains the negligible plasma concentrations of indoxyl sulfate in healthy individuals."

The researchers conclude that this remote sensing and signalling pathway is a homeostatic mechanism that regulates the levels of microbial metabolites by controlling their secretion by the kidney. "In the early phases of kidney disease it may be possible to influence kidney function," says Masereeuw. "By supporting the renal secretion of harmful endogenous waste products, we might find a way to attenuate progression of this disease."

Ellen F. Carney

ORIGINAL ARTICLE Jansen, J. et al. Remote sensing and signaling in kidney proximal tubules stimulates gut microbiome-derived organic anion secretion. Proc. Natl Acad. Sci. USA 116, 16105-16110 (2019) 